\title{
Cytidine 5'-diphosphocholine ameliorates hyperoxic lung injury in a neonatal rat model
}

\author{
Merih Cetinkaya', Mehmet Cansev², Ilker M. Kafa ${ }^{3}$, Cuneyt Tayman', Ferhat Cekmez', Fuat Emre Canpolat', Turan Tunc and \\ S. Umit Sarici'
}

BACKGROUND: Bronchopulmonary dysplasia (BPD) is an important cause of morbidity. The aim of this study was to evaluate the preventive effect of cytidine $5^{\prime}$-diphosphocholine (CDP-choline) treatment on hyperoxic lung injury in a neonatal rat model.

METHODS: A total of 30 newborn pups were divided into control, hyperoxia, and hyperoxia + CDP-choline groups. After birth, pups in the control group were kept in room air and received saline injections, whereas those in hyperoxia and hyperoxia + CDP-choline groups were exposed to $95 \% \mathrm{O}_{2}$ and received daily injections of saline and CDP-choline throughout postnatal day 10, respectively. Histopathological scoring, radial alveolar count, lamellar body membrane protein expression, fibrosis, proinflammatory cytokine levels, lung tissue and bronchoalveolar lavage (BAL) fluid phospholipid content, and apoptosis were evaluated.

RESULTS: Hyperoxia-induced severe lung damage was reduced significantly by CDP-choline treatment. Radial alveolar count and lamellar body membrane protein expression were significantly recovered, and the number of terminal deoxynucleotidyl transferase-mediated deoxyuridine triphosphate nick-end labeling-positive cells, active caspase-3 expression, and tissue proinflammatory cytokine levels were decreased by CDP-choline administration. Lung tissue and BAL phospholipid contents showed significant increases after CDP-choline administration.

CONCLUSION: These data show that CDP-choline ameliorates hyperoxic lung injury in a neonatal rat model. It may therefore be suggested that CDP-choline may be a novel therapeutic option for the prevention of BPD.

B ronchopulmonary dysplasia (BPD) is an important cause of morbidity in preterm infants that results in prolonged hospitalization, discharge from hospital with oxygen therapy, frequent readmission to the hospital with respiratory problems, and increased neurodevelopmental risk (1). Although BPD has a multifactorial etiology, major risk factors for BPD development include preterm birth, lower gestational age and birth weight, need for supplemental oxygen and ventilatory support, oxygen toxicity, decreased host antioxidant defenses, patent ductus arteriosus, and pre- and postnatal infections (2). Therefore, treatment of evolving BPD may be challenging due to the complex balance between these contributing risk factors. Efficacy of parenteral administration of vitamin A or caffeine, the two successful treatments developed to prevent BPD, was supported by evidence from randomized, controlled studies (3), whereas many other treatments aiming at preventing BPD have failed to demonstrate a reduction in BPD rates (2). Therefore, newer strategies and pharmacological approaches to prevent BPD are required in order to decrease $\mathrm{BPD}$ incidence and severity.

Cytidine 5'-diphosphocholine (CDP-choline), which is composed of cytidine and choline linked by a diphosphate bridge, is an endogenous intermediate in the synthesis of the major membrane phospholipid, phosphatidylcholine (PC) (4). PC is quantitatively the most important phospholipid, accounting for $70-85 \%$ of the total surfactant phospholipids, which are major constituents of surfactant lipids (5). The primary function of surfactant is to decrease surface tension at the air-liquid surface in the alveoli and distal bronchioli, to promote lung expansion during inspiration in order to prevent atelectasis at end expiration. It also plays an important role in pulmonary host defense and local immunomodulation by enhancing the stability of the film that floats on the alveolar linings, facilitating mucociliary transport, exhibiting antioxidant activity with antibacterial/antiviral properties (5). Due to persisting surfactant abnormalities including an ongoing quantitative deficiency in surfactant components, decreased function of endogenous surfactant, and increased surfactant turnover in ventilated preterms with evolving BPD, surfactant therapy was suggested to show benefits in prevention of BPD (6). In addition, the beneficial effects of additional surfactant therapy for prevention of BPD were also evaluated (7).

In addition, in a few studies with conflicting outcomes, CDP-choline was administered parenterally at a dose range of $100-300 \mathrm{mg} / \mathrm{kg} / \mathrm{d}$ to preterm infants to increase the synthesis of lung phospholipids for alleviating the symptoms of respiratory distress syndrome $(8,9)$. However, to the best of our knowledge, no study evaluated the efficacy of CDP-choline for preventing BPD. Therefore, the aim of this experimental study was to investigate the possible protective effect of CDP-choline against hyperoxic lung injury in a neonatal rat model. 


\section{RESULTS}

No significant difference was detected between mean birth weights of pups in the control, hyperoxia, and hyperoxia + CDP-choline groups $(5.0 \pm 0.4$ vs. $4.9 \pm 0.2$ vs. $5.0 \pm 0.3 \mathrm{~g}$, respectively) $(P>0.05)$. However, the mean body weight of pups in the hyperoxia + CDP-choline group $(15.2 \pm 1.2 \mathrm{~g})$ was significantly greater than that of pups in the hyperoxia group $(12.2 \pm 0.9 \mathrm{~g})(P=0.02)$, but not in the control group $(17.4 \pm 1.6 \mathrm{~g} ; P>0.05)$ at end of the study (postnatal day $(\mathrm{P}) 10)$. During the experimental procedures, two pups in the hyperoxia group and one pup in the hyperoxia + CDP-choline group died. However, no significant difference was found between these two groups in terms of survival $(P>0.05)$.

Severity of lung damage was evaluated between grades 1 and 4 by histopathologic examination (Figures 1a-c and 2). Thickening of the alveolar septi or cell infiltration was not observed in the control and hyperoxia + CDP-choline groups. CDP-choline treatment significantly improved histological grading of lung injury as compared with saline treatment in the hyperoxia group $(P=0.01)$ (Figure 1a-c). Masson's trichrome stained sections also showed cell infiltration, edema, and fibrosis in the hyperoxia group, which were not noticeably observed in the control and hyperoxia + CDP-choline groups (Figure 1d-f). Radial alveolar count, reflected by the number of intact alveoli, was also significantly greater in the hyperoxia + CDP-choline group as compared with the hyperoxia group $(P<0.05)$ (Figure 2$)$.
Although expression of P180 lamellar body membrane protein was decreased in the hyperoxia group, it was recovered by CDPcholine treatment (Figures $1 \mathrm{~g}-\mathbf{i}$ and 2 ). By contrast, the number of terminal deoxynucleotidyl transferase-mediated deoxyuridine triphosphate nick-end labeling (TUNEL)-positive cells per unit area (Figure 3) and active caspase-3 expression (209.2 $\pm 13.7 \%$ in the hyperoxia group vs. $127.9 \pm 5.6 \%$ in the hyperoxia + CDPcholine group) (Figure 4) were lower in the hyperoxia + CDPcholine group as compared with those in the hyperoxia group $(P$ $<0.05)$. Levels of $\beta$-actin, the protein that was used as a proteinloading control for gel electrophoresis, did not change between the groups.

To further investigate the possible mechanism of action of CDP-choline treatment, lung tissue inflammatory cytokine levels were also analyzed. In the hyperoxia group, levels of interleukin (IL) $-1 \beta$, IL-6, and tumor necrosis factor- $\alpha$ (TNF- $\alpha$ ) were found to be increased as compared with those of the control group ( $P$ $<0.05$ ), whereas CDP-choline treatment significantly reduced hyperoxia-associated elevations of IL-1 $\beta$ (from 148.9 \pm 12.4 to $112.1 \pm 9.4 \mathrm{pg} / \mathrm{ml}$ ), IL-6 (from $43.7 \pm 4.5$ to $28.6 \pm 4.1 \mathrm{pg} / \mathrm{ml}$ ), and TNF- $\alpha$ (from $246.7 \pm 30.9$ to $146.2 \pm 20.7 \mathrm{pg} / \mathrm{ml})(P<0.05)$ (Table 1), suggesting an anti-inflammatory effect of CDP-choline treatment.

To investigate the effect of supplemented CDP-choline on surfactant components, a phospolipid assay was performed in bronchoalveolar lavage (BAL) fluid and lung tissue homogenates. As

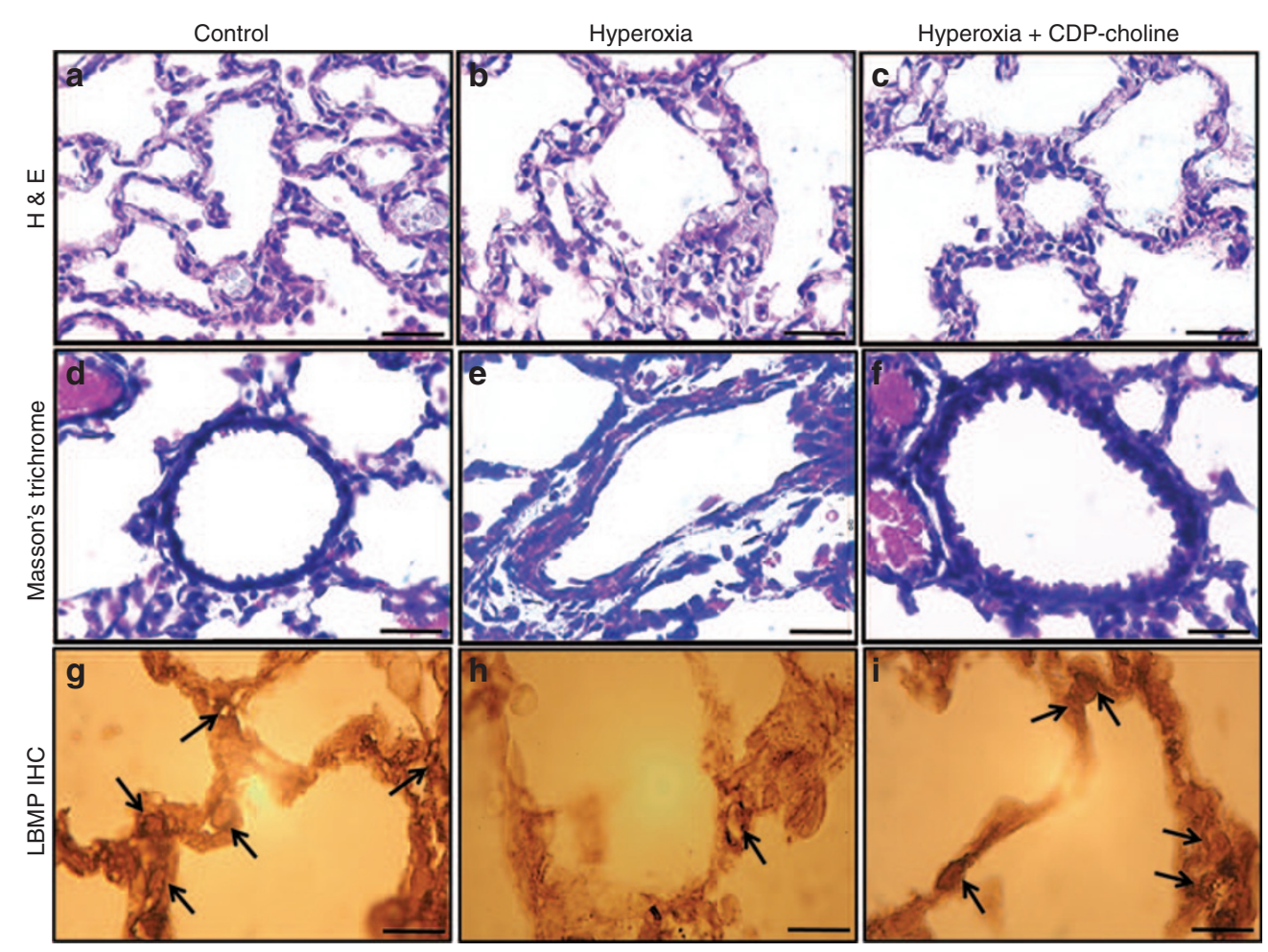

Figure 1. Representative images of (a-c) hematoxylin-eosin staining, (d-f) Masson's trichrome staining, and (g-i) immunohistochemistry for lamellar body membrane protein. Histological appearance of the lungs in the hyperoxia group is characterized by partial destruction of the lung tissue (in $\mathbf{b}$ ), cell infiltration, edema, and fibrosis (in b and e), which are not notably observed in the control (in $\mathbf{a}$ and $\mathbf{d}$ ) and hyperoxia + CDP-choline (in c and f) groups. (g-i) Abundant positive immunohistochemistry for the lamellar body membrane protein for control and hyperoxia + CDP-choline groups (black arrows, DAB-positive lamellar body membrane protein immunoreactivities). Bars, $50 \mu \mathrm{m}$ (in d-f); $25 \mu \mathrm{m}$ (in a-c and g-i). CDP-choline, cytidine $5^{\prime}$-diphosphocholine; DAB, 3,3'-diaminobenzidine; H\&E, hematoxylin-eosin staining; LBMP IHC, lamellar body membrane protein immunohistochemistry. 


\section{Articles | Cetinkaya et al.}

a

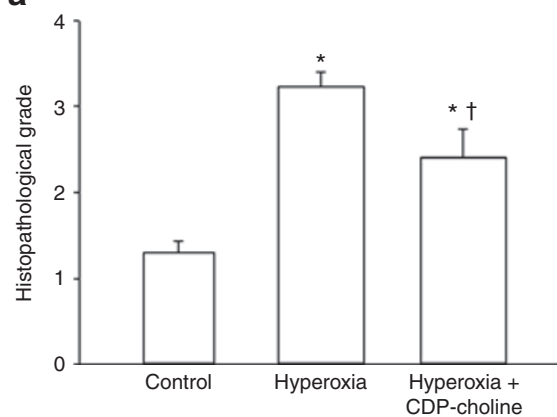

b

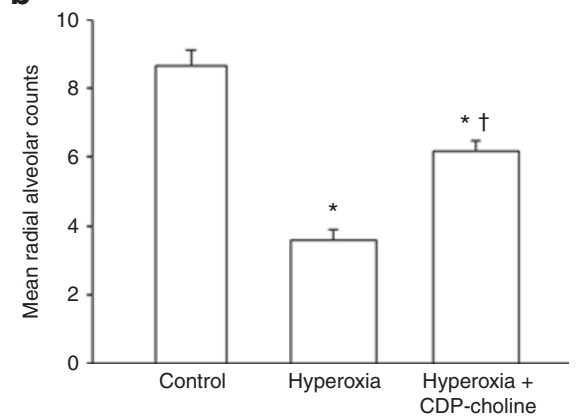

C

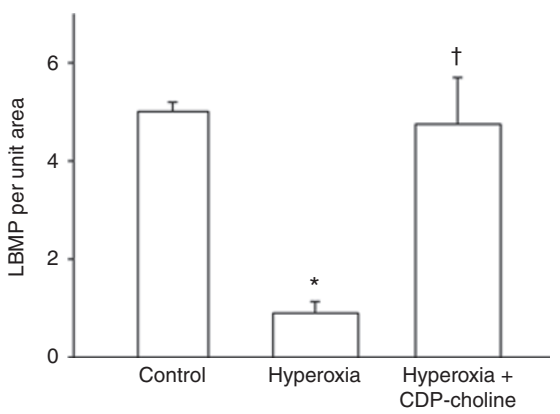

Figure 2. Bar graphs for (a) histopathologic evaluation, (b) radial alveolar count, and (c) lamellar body membrane protein expression. ${ }^{*} P<0.05$, significantly different from control group; ${ }^{\dagger} P<0.05$, significantly different from hyperoxia group using one-way ANOVA followed by post hoc Holm-Sidak test. CDP-choline, cytidine 5'-diphosphocholine; LBMP, lamellar body membrane protein.

a

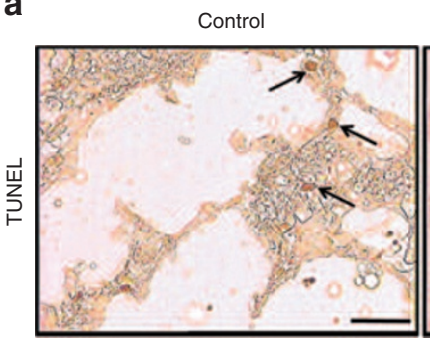

Hyperoxia

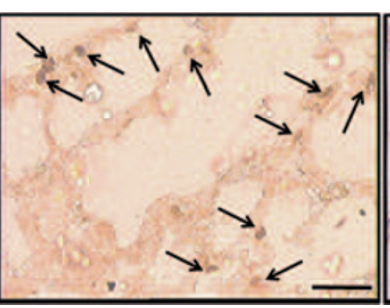

Hyperoxia + CDP-choline

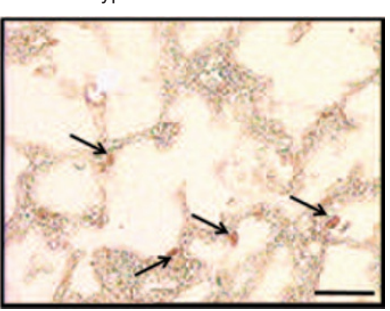

b

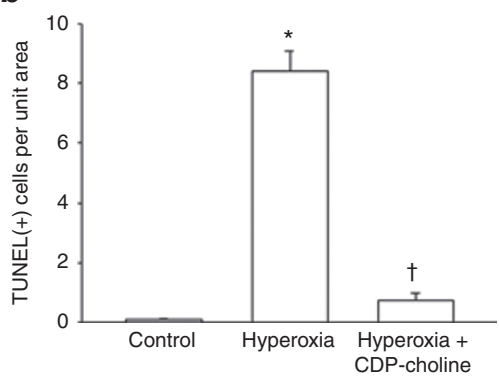

Figure 3. TUNEL-positive cell counts. (a) Immunohistochemistry images and (b) bar graph for TUNEL-positive cell counts. Arrows indicate TUNELpositive cells. ${ }^{*} P<0.05$, significantly different from control group; ${ }^{\dagger} P<0.05$, significantly different from hyperoxia group using one-way ANOVA followed by post hoc Holm-Sidak test. Bar, $25 \mu \mathrm{m}$. CDP-choline, cytidine 5'-diphosphocholine; TUNEL, terminal deoxynucleotidyl transferase-mediated deoxyuridine triphosphate nick-end labeling.
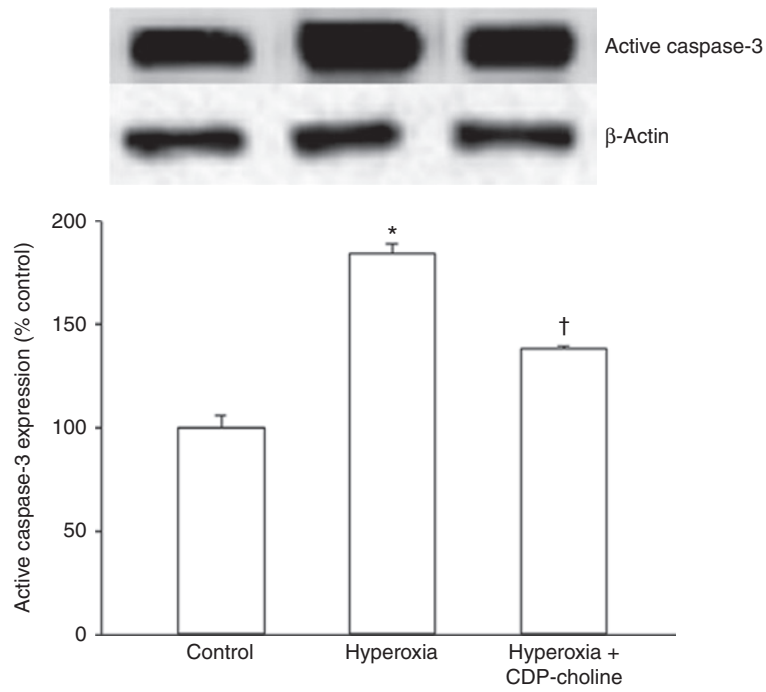

Figure 4. Bar graph showing the effect of CDP-choline treatment on active caspase-3 expression in hyperoxic lung injury. ${ }^{*} P<0.05$, significantly different from control group; ${ }^{\dagger} P<0.05$, significantly different from hyperoxia group using one-way ANOVA followed by post hoc Holm-Sidak test. $\beta$-Actin was used as a protein-loading control. CDP-choline, cytidine 5 -diphosphocholine.

shown in Table 2, the content of total phospholipids and phospholipid classes including PC, phosphatidylglycerol, and disaturated PC (DSPC) significantly decreased in both BAL and lung tissue samples of rat pups exposed to hyperoxia as compared with those in control pups, whereas CDP-choline treatment significantly increased the contents of the measured phospholipid classes (Table 2).

In addition, biochemical analyses revealed that subjection to hyperoxia significantly decreased tissue superoxide dismutase (SOD) and glutathione peroxidase (GSH-Px) activities, whereas it increased tissue malondialdehyde (MDA) content and myeloperoxidase (MPO) activity (Table 3). By contrast, mean tissue SOD and GSH-Px activities were significantly higher in the hyperoxia + CDP-choline group as compared with the hyperoxia group $(95.6 \pm 6.1$ vs. $63.7 \pm 2.5 \mathrm{U} / \mathrm{mg}$ protein, $P=0.001$ and $11.5 \pm 1.5$ vs. $5.6 \pm 1.8 \mathrm{U} / \mathrm{g}$ protein, $P=$ 0.01 , respectively), indicating the efficacy of CDP-choline on decreasing oxidative stress. Mean tissue MDA content and MPO activity $(52.9 \pm 3.2 \mathrm{vs.} 68.8 \pm 7.4 \mathrm{nmol} / \mathrm{g}$ protein, $P=0.001$ and $55.5 \pm 6.8$ vs. $76.2 \pm 2.1 \mathrm{U} / \mathrm{g}$ protein, $P=0.001$, respectively) were significantly lower in the hyperoxia $+\mathrm{CDP}$-choline group as compared with those in the hyperoxia group, suggesting a CDP-choline effect on decreasing lipid peroxidation (Table 3 ).

\section{DISCUSSION}

These data show that CDP-choline treatment improves histopathological score, decreases fibrosis, improves alveolarization by preserving radial alveolar count and lamellar body protein expression in the alveoli, reduces TUNEL-positive cell counts 
Table 1. Levels of cytokines in lung homogenates

\begin{tabular}{|c|c|c|c|}
\hline $\begin{array}{l}\text { Cytokine levels, } \\
\mathrm{pg} / \mathrm{ml}\end{array}$ & Control group & $\begin{array}{l}\text { Hyperoxia } \\
\text { group }\end{array}$ & $\begin{array}{l}\text { Hyperoxia + } \\
\text { CDP-choline } \\
\text { group }\end{array}$ \\
\hline IL-1 $\beta$ & $91.2 \pm 8.7$ & $148.9 \pm 12.4^{* *}$ & $112.1 \pm 9.4^{* * *}$ \\
\hline IL-6 & $24.4 \pm 3.2$ & $43.7 \pm 4.5^{*}$ & $28.6 \pm 4.1^{* * *}$ \\
\hline TNF- $\alpha$ & $104.0 \pm 20.3$ & $246.7 \pm 30.9^{* *}$ & $146.2 \pm 20.7^{* * *}$ \\
\hline
\end{tabular}

Table 2. Lung tissue and bronchoalveolar lavage fluid phospholipid levels in the study groups

\begin{tabular}{|c|c|c|c|}
\hline & Control & Hyperoxia & $\begin{array}{l}\text { Hyperoxia + } \\
\text { CDP-choline }\end{array}$ \\
\hline \multicolumn{4}{|c|}{ Lung tissue phospholipids (nmol/mg protein) } \\
\hline Total phospholipids & $298.5 \pm 6.4$ & $220.8 \pm 5.4^{* *}$ & $271.8 \pm 1.5^{+}$ \\
\hline PC & $146 \pm 2.7$ & $107.5 \pm 3.2^{* *}$ & $130 \pm 1^{+}$ \\
\hline DSPC & $47.5 \pm 1.2$ & $35.5 \pm 1^{* *}$ & $42.5 \pm 1^{\dagger}$ \\
\hline PG & $9.5 \pm 0.2$ & $7.1 \pm 0.2^{*}$ & $8.1 \pm 0.2^{* * *}$ \\
\hline \multicolumn{4}{|c|}{ BAL fluid phospholipids (nmol/ml) } \\
\hline Total phospholipids & $31 \pm 1.6$ & $20.5 \pm 0.6^{* *}$ & $28.5 \pm 0.7^{+}$ \\
\hline PC & $24.3 \pm 0.5$ & $17.9 \pm 0.5^{* *}$ & $21.7 \pm 0.2^{\dagger}$ \\
\hline DSPC & $15.8 \pm 0.4$ & $11.8 \pm 0.3^{* *}$ & $14.2 \pm 0.1^{\dagger}$ \\
\hline PG & $3.2 \pm 0.1$ & $2.4 \pm 0.1^{*}$ & $2.7 \pm 0.1^{* * *}$ \\
\hline
\end{tabular}

BAL, bronchoalveolar lavage; DSPC, disaturated phosphatidylcholine; PC, phosphatidylcholine; $\mathrm{PG}$, phosphatidylglycerol.

${ }^{*} P<0.05 ;{ }^{* *} P<0.01$ compared with control group; ${ }^{* *} P<0.05 ;{ }^{*} P<0.01$ compared with hyperoxia group using one-way ANOVA followed by post hoc Holm-Sidak test.

Table 3. Lung tissue malondialdehyde (MDA) content and superoxide dismutase (SOD), glutathione peroxidase (GSH-Px), and myeloperoxidase (MPO) activities

\begin{tabular}{|c|c|c|c|}
\hline Biochemical analyses & $\begin{array}{c}\text { Control } \\
\text { group, } n=10\end{array}$ & $\begin{array}{c}\text { Hyperoxia } \\
\text { group, } n=10\end{array}$ & $\begin{array}{c}\text { Hyperoxia + } \\
\text { CDP-choline } \\
\text { group, } \\
n=10\end{array}$ \\
\hline MDA (nmol/g protein) & $31.1 \pm 3.7$ & $68.8 \pm 7.4^{* *}$ & $52.9 \pm 3.2^{* * *}$ \\
\hline SOD (U/mg protein) & $114.4 \pm 9.2$ & $63.7 \pm 2.5^{* *}$ & $95.6 \pm 6.1^{\dagger}$ \\
\hline GSH-Px (U/mg protein) & $12.5 \pm 1.4$ & $5.6 \pm 1.8^{*}$ & $11.5 \pm 1.5^{* * *}$ \\
\hline MPO (U/g protein) & $36.5 \pm 2.3$ & $76.2 \pm 2.1^{* *}$ & $55.5 \pm 6.8^{+}$ \\
\hline
\end{tabular}

and active caspase- 3 expression, increases antioxidant activity, and decreases lipid peroxidation and inflammation in the injured lung tissues of neonatal rats. In addition, both lung tissue and BAL fluid phospholipid contents were significantly increased by CDP-choline therapy. To the best of our knowledge, this is the first experimental study that reports amelioration of hyperoxic lung injury by CDP-choline treatment in a neonatal rat model.

Preterm infants and their lungs are susceptible to injury as a result of resuscitation, mechanical ventilation, oxygen therapy, and surfactant deficiency (10). BPD is characterized by decreased alveolar and vascular lung development resulting in enlarged alveoli and is complicated by inflammation, abnormal coagulation, fibrinolysis, oxidative stress, and, at later stages, pulmonary hypertension (10). Neonatal exposure to hyperoxia in animals was reported to result in progressive lung disease that strongly resembles BPD in premature infants (11). Therefore, we used this experimental BPD model, which induces lung injury by exposure to hyperoxia.

Hyperoxic injury caused both apoptotic and necrotic cell death in an adult murine model, and the number of apoptotic cells in the lung exposed to hyperoxia correlated with the degree of the injury (12). High concentrations of oxygen also resulted in apoptosis in the lungs in a neonatal mouse model in which the degree of apoptosis was found to be associated with the duration of hyperoxia and the degree of lung injury (13). Therefore, the authors suggested that apoptosis due to hyperoxia during the crucial period of lung development might adversely affect future lung growth. In accordance with these studies, our data showed that CDP-choline significantly decreased the number of TUNEL-positive cells and active caspase-3 expression in hyperoxic lung injury.

Although BPD has a multifactorial etiology, pre- and postnatal infection and inflammation are the major contributors to its pathogenesis (2). Several studies showed increased protein levels and high mRNA expression of proinflammatory cytokines in airway secretions and bronchoalveolar cells of premature infants who developed BPD $(14,15)$. We also observed in the current study that proinflammatory cytokines TNF- $\alpha$, IL- 6 , and IL- $1 \beta$ were increased due to exposure to hyperoxia in the lungs of neonatal rats. However, CDP-choline treatment significantly reduced the levels of these proinflammatory cytokines. CDP-choline is a choline donor because part of exogenously administered CDPcholine is metabolized to choline in the circulation (16). Choline is an essential nutrient and a precursor of the neurotransmitter acetylcholine (ACh) as well as various membrane phospholipids (17). Choline may have anti-inflammatory effects by activating $\alpha-7$ nicotinic ACh receptors (18). In agreement, choline administration was shown to reduce the elevation of circulating TNF- $\alpha$ levels and attenuate endotoxin-induced multiple organ injury (19), whereas choline deficiency results in increased levels of proinflammatory cytokines such as TNF- $\alpha$ (20). Because exogenously administered CDP-choline is a source of choline (16), our observation that CDP-choline reduces tissue levels of proinflammatory cytokines might have resulted from the anti-inflammatory action of choline (through stimulation of $\alpha-7$ nicotinic ACh receptors) that derived from CDP-choline metabolism.

In addition, because choline is a precursor for the synthesis of ACh both in the brain and periphery, including nonneuronal cells in the respiratory tract (21), choline metabolized from CDP-choline might have enhanced ACh synthesis and cholinergic activity in our study. Recent findings suggest that through the cholinergic anti-inflammatory pathway (22), ACh regulates additional functions in the airways, including inflammation and remodeling during inflammatory airway diseases $(23,24)$. Therefore, because CDP-choline administration has 


\section{Articles $\mid$ Cetinkaya et al.}

been shown to exhibit body responses of a cholinergic nature (25), the anti-inflammatory effects of CDP-choline might have resulted from enhanced ACh synthesis and cholinergic activity.

Another mechanism by which CDP-choline prevented BPD in our study might include counteraction of cytokine effects on cellular membranes by CDP-choline. Exogenously administered CDP-choline enhances membrane phospholipid levels in the brain and lungs (8). Therefore, as a building block of PC, the major constituent of surfactant, CDP-choline might have shown benefit by enhancing surfactant synthesis in our study. Surfactant therapy was suggested to confer benefit in the prevention of BPD in previous studies that demonstrated persisting surfactant abnormalities including an ongoing quantitative deficiency in surfactant components (especially surfactant proteins), decreased function of endogenous surfactant for lowering surface tension, and increased surfactant turnover in ventilated preterms evolving BPD (6). In addition, expression of surfactant proteins $\mathrm{A}, \mathrm{B}$, and $\mathrm{C}$ were found to be altered in animal models of chronic exposure to high oxygen concentrations (26). The relative deficiencies of surfactant proteins were reported to be indicators of BPD for lower lung volume and infection in evolving BPD in a baboon model of BPD (27). In another study, surfactant function and contents of surfactant proteins were shown to be altered in mechanically ventilated preterm infants (6). In accordance with these data, temporary improvement in pulmonary function of preterm infants who required prolonged mechanical ventilation was achieved with exogenous surfactant supplementation (28), suggesting surfactant's preventive effect on BPD development (7). Hence, we analyzed both lung tissue and BAL fluid phospholipid concentrations and showed that the phospholipid classes PC, phosphatidylglycerol, and DSPC reached near-control levels after CDP-choline administration. Therefore, as a precursor of PC and surfactant synthesis, CDP-choline might have ameliorated the lung damage caused by BPD in our study by reducing membrane breakdown and enhancing phospholipid and, possibly, surfactant production.

Oxygen toxicity, which has been suggested as a significant contributing factor for BPD development in premature infants (2), is associated with generation of hydroxyl radicals and peroxidation of membrane lipids. It is well known that proinflammatory cytokines activate phospholipase $\mathrm{A}_{2}$ and PC-phospholipase C in order to hydrolize PC (4). PC hydrolysis by phospholipase $\mathrm{A}_{2}$ results in the release of lyso-PC, an inhibitor of CTP-phosphocholine cytidylyltransferase enzyme, which is involved in PC synthesis, and free fatty acids including arachidonic acid, which is a significant source of reactive oxygen species. CDP-choline was previously reported to prevent membrane destruction, decrease free radical generation, and preserve the natural mechanisms against oxidative stress, particularly in hypoxia-ischemia models (4). In agreement with these observations, our data indicate that CDP-choline treatment preserves GSH-Px and SOD activities as well as reducing tissue MDA content and MPO activity, suggesting that one of the mechanisms by which CDP-choline decreases hyperoxic lung injury is enhancement of antioxidant activity and reduction of lipid peroxidation in a neonatal rat model of BPD. It may also suggest that the effect of CDP-choline on apoptosis might have resulted from attenuation of oxidative stress by restoration of antioxidant enzyme levels.

A number of previous studies evaluated surfactant kinetics in preterm infants who did or did not develop BPD. One study reported that tracheal aspirate DSPC concentration decreased significantly in infants who subsequently developed BPD (29). The loss of DSPC palmitate from the alveolar pool was significantly higher, whereas recycling of DSPC palmitate through the intracellular pool was significantly lower (30). Therefore, our findings regarding the increased BAL fluid and lung tissue phospholipid contents after CDP-choline administration might explain the possible role of CDP-choline in the attenuation of hyperoxic lung injury in our experimental setting.

To the best of our knowledge, two previous human studies evaluated CDP-choline treatment in preterm infants with respiratory distress syndrome $(8,9)$. Although parenteral CDP-choline administration showed no beneficial effects at a $100 \mathrm{mg} / \mathrm{kg}$ dosage for $7 \mathrm{~d}$ (9), it was reported to reduce the severity of respiratory distress and oxygen requirement when it was administered at a $300 \mathrm{mg} / \mathrm{kg}$ dosage for a longer duration (8). Therefore, a higher dose and a longer duration of CDPcholine administration might explain the beneficial effect of CDP-choline against hyperoxic lung injury in our study and suggests its applicability in newborns with BPD.

In conclusion, our study is the first to show the efficacy of CDP-choline treatment in hyperoxic lung injury in a neonatal rat model by several mechanisms including enhancement of phospholipid production as well as reduction of fibrosis, apoptosis, and lipid peroxidation. These findings might provide new insights into the prevention of BPD development by CDP-choline treatment in premature infants. Nevertheless, our study is limited by the lack of dose and time courses of CDP-choline effects, and future studies are warranted to further confirm its effects and reveal the mechanism of action of CDP-choline treatment in hyperoxic lung injury and BPD.

\section{METHODS}

\section{Animals and Experimental Design}

This study was approved by the Experimental Animal Ethics Committee of Gulhane Military Medical Academy (Ankara, Turkey). In addition, the guidelines provided by the US National Institutes of Health (Bethesda, MD) for the care and use of laboratory animals were followed.

Sprague Dawley rats with dated pregnancies were housed in individual cages with free access to water and laboratory chow. Pups born by spontaneous delivery to four time-mated full-term Sprague Dawley pregnant rats were pooled, randomized, and delivered back to nursing dams. A total of 30 newborn pups were divided equally into three groups as follows: control group (composed of pups subjected to room air containing $21 \% \mathrm{O}$ and receiving saline), hyperoxia group (composed of pups subjected to $95 \% \mathrm{O}_{2}$ and receiving saline), and hyperoxia + CDP-choline group (composed of pups subjected to $95 \%$ $\mathrm{O}_{2}$ and receiving $300 \mathrm{mg} / \mathrm{kg} / \mathrm{d}$ CDP-choline).

Experiments began immediately after birth and continued throughout P10. Nursing dams were rotated between hyperoxiaexposed and room air-exposed litters every $24 \mathrm{~h}$ to prevent oxygen toxicity in the dams. In pups subjected to hyperoxia, continuous $95 \%$ $\mathrm{O}_{2}$ exposure was achieved in a Plexiglas chamber $(70 \times 60 \times 30 \mathrm{~cm})$ by 
a flow-through system. The oxygen level inside the Plexiglas chamber was monitored continuously with a Ceramatec oxygen analyzer (MAXO2; Ceramatec, Salt Lake City, UT) to maintain $\geq 95 \% \mathrm{O}_{2}$ saturation. $\mathrm{CO}_{2}$ was kept below $0.5 \%$ using a gas monitor (Apex; BW Technologies, Lincolnshire, IL). Temperature and humidity were maintained at $22-25^{\circ} \mathrm{C}$ and $60-70 \%$, respectively.

Intraperitoneal injections of saline $(4 \mathrm{ml} / \mathrm{kg})$ in the control and hyperoxia groups and those of CDP-choline $(300 \mathrm{mg} / \mathrm{kg})$ in the hyperoxia + CDP-choline group were performed daily from the first day of life (P1) through P10, when the study was terminated. The pups in each group were weighed daily using a sensitive scale of $0.01 \mathrm{~g}$, and their weights were recorded.

\section{Lung Tissue Procurement}

Pups were killed at P10 under deep anesthesia by intraperitoneal injection of a combination of ketamine $(100 \mathrm{mg} / \mathrm{kg}$, Ketalar; Zentiva Saglik Urunleri, Kirklareli, Turkey) and xylazine $(10 \mathrm{mg} / \mathrm{kg}$, Xylazine Bio 2\%; Bioveta, Ivanovice na Hane, Czech Republic). The thorax was opened and the lungs were exposed. Right lungs of rat pups were excised and weighed immediately for analyses of tissue cytokine levels, active caspase-3 expression, phospholipid content, and oxidant/ antioxidant enzyme activities and MDA content, whereas left lungs were obtained after ligation of the right main bronchus and perfusion with a fixation solution for histopathologic and immunohistochemical evaluation.

\section{Histopathologic and Immunohistochemical Evaluation of the Lungs}

Left lungs were perfused with $0.1 \mathrm{~mol} / 1$ phosphate-buffered saline (PBS) containing 4\% paraformaldehyde under a constant inflation pressure of $5 \mathrm{~cm} \mathrm{H}_{2} \mathrm{O}$ maintained via a tracheal catheter. On completion of perfusion, the trachea was ligated with a surgical suture, and the lungs were incubated in fresh $4 \%$ paraformaldehyde-PBS solution on ice for 4-5h. The perfusion solution was then replaced with two quick changes of cold PBS to remove exterior debris. The lungs were transferred to a filtered sterile PBS/30\% sucrose solution and stored at $4{ }^{\circ} \mathrm{C}$ until fully equilibrated. The lungs were then paraffin embed$\mathrm{ded}$, and these paraffin blocks were sliced into $5-\mu \mathrm{m}$ sections. Sections were chosen according to a systematic random sampling procedure and then mounted onto poly-L-lysine-coated slides (Histobond adhesion slides; Paul Marienfeld, Lauda-Konigshofen, Germany).

Slides were stained with standard hematoxylin-eosin and Masson's trichrome techniques for histopathologic evaluations and with ABC technique for the lamellar body membrane protein. For immunohistochemical detection of lamellar body membrane protein, sections were subjected to dehydration following treatment with 3\% hydrogen peroxide for $30 \mathrm{~min}$. After nonspecific blocking with goat serum for $30 \mathrm{~min}$, the sections were incubated overnight with primary antibody against P180 lamellar body protein (Abcam, Cambridge, MA) at $4{ }^{\circ} \mathrm{C}$, followed by treatment with biotinylated antimouse secondary antibody (1:200; Vector Laboratories, Peterborough, UK) for $30 \mathrm{~min}$ at room temperature. Following the avidin-biotin complex treatment, 3,3'-diaminobenzidine (Vector Laboratories) was used for color development. Negative control slides, omitting the primary antibody, were also included. The tissue sections were examined using a light microscope equipped with a digital camera. Positive cells per unit area were counted five times on $25 \mu \mathrm{m}$ apart sections, 10 sections for each animal. Histopathologic examination and immunohistochemical scoring were performed in a blinded manner by the same investigator. Histopathologic grading was done as follows: grade 1, normal histology; grade 2, moderate leukocytic infiltration; grade 3, leukocytic infiltration, edema, and partial destruction; and grade 4, total destruction of the tissue as described previously (31). Radial alveolar count for the assessment of alveolar development was performed on the digital images of sections. Briefly, a line was superimposed from a center of a respiratory bronchiole to the nearest connective tissue septum at right angles to the epithelium, and the number of alveolar septi crossed by this line was counted on 3-4 sections for each animal (32).

Apoptosis was evaluated by TUNEL technique using an in situ cell death detection POD kit (Roche Molecular Biochemicals, Mannheim, Germany). Sections were chosen according to a systematic random sampling procedure and then mounted onto poly-L-lysine-coated slides (Histobond adhesion slides; Paul Marienfeld). Sections were heated at $60^{\circ} \mathrm{C}$ for $45 \mathrm{~min}$, followed by washing in xylene and rehydration through a graded series of alcohol (ethanol 100, 95, 80, and 70\%), and washed in distilled water. They were then incubated in $20 \mu \mathrm{g} / \mathrm{ml}$ proteinase K solution (Roche Applied Sciences, Penzberg, Germany) for $20 \mathrm{~min}$ at room temperature and immersed in $3 \% \mathrm{H}_{2} \mathrm{O}_{2}$ in methanol for $10 \mathrm{~min}$. Permeabilization with Triton X (Sigma-Aldrich, St Louis, MO) for $10 \mathrm{~min}$ was performed on ice. The sections were then incubated with TUNEL reaction mixture at $37^{\circ} \mathrm{C}$ in a humidified chamber for $1 \mathrm{~h}$ followed by converter-peroxidase treatment at 37 ${ }^{\circ} \mathrm{C}$ for $30 \mathrm{~min}$. Diaminobenzidine tetrahydrochloride (diaminobenzidine/metal concentrate:peroxidase buffer; Roche Applied Sciences) was used as the chromogene, and the slides were counterstained with hematoxylin (Harris's hematoxylin; Sigma-Aldrich) and coverslipped (DPX mountant; Fluka, St Louis, MO) for light microscopy.

\section{Active Caspase-3 Expression}

Right lungs were homogenized by 50 -fold dilution (wt/vol) in icecold PBS (pH 7.2) using a homogenizer (T18 basic Ultra-Turrax; IKA, Staufen, Germany). Aliquots of homogenates were used for total protein analysis by bicinchoninic acid assay (Thermo Fisher Scientific, Rockford, IL). Aliquots of homogenates were mixed with equal volumes of Laemmi loading buffer and boiled for $5 \mathrm{~min}$ before gel electrophoresis. Equal amounts of protein were loaded and separated using sodium dodecyl sulfate-polyacrylamide gel electrophoresis (4-20\%; Bio-Rad, Hercules, CA). Proteins were then transferred onto polyvinylidene fluoride membranes (Immobilon-P; Millipore, Billerica, MA). The remaining binding sites were blocked with $4 \%$ nonfat dry milk (Carnation, Glendale, CA) for $30 \mathrm{~min}$ in Tris-buffered saline with Tween (TBST). Membranes were then rinsed five times in TBST buffer and incubated overnight in TBST solution containing the primary antibody against active caspase-3 (Abcam) and $\beta$-actin (Abcam), which was used as a loading control for lung homogenates. Following overnight incubation and five rinses in TBST buffer, blots were incubated for $1 \mathrm{~h}$ with the appropriate peroxidase-linked secondary antibody (GE Healthcare, Waukesha, WI). Blots were then rinsed in TBST buffer five times, and protein-antibody complexes were detected and visualized using the enhanced chemiluminescence system (GE Healthcare) and developed on Kodak X-AR film (Kodak Turkiye, Istanbul, Turkey). Films were digitized using a Supervista S-12 scanner with a transparency adapter (UMAX Technologies, Freemont, CA). Immunoreactive bands were compared densitometrically using the public domain NIH Image program available on the Internet at http://rsbweb.nih.gov/ij/ download.html. Areas under the absorbance curve were expressed as arbitrary units and normalized as percentages of those generated in the same blot using samples from lungs of control animals.

\section{Measurement of Lung Tissue Cytokine Content}

Aliquots of homogenates were centrifuged at $12,000 \mathrm{~g}$ for $20 \mathrm{~min}$ at $4{ }^{\circ} \mathrm{C}$, and cell-free supernatants were used for determining lung tissue proinflammatory cytokine (TNF- $\alpha$, IL-6, and IL-1 $\beta$ ) contents by specific enzyme-linked immunosorbent assay kits (R\&D Systems, Minneapolis, MN) as described previously (33).

\section{BAL Fluid Collection}

In separate experiments, rat pups anesthetized with intraperitoneal ketamine plus xylazine were placed in a supine position, and a cannula was inserted into the trachea. Ice-cold isotonic saline $(1 \mathrm{ml})$ was gently instilled with a syringe into the lung via the trachea and then withdrawn 10 times. Recovered BAL fluid was immediately centrifuged at 5,000 rpm for $10 \mathrm{~min}$ to remove cells, and samples from four pups from each treatment group were pooled.

\section{Phospholipid Assay}

Phospholipids were extracted from lung tissue homogenates and BAL fluid using a chloroform-methanol-water mixture (2:1:1 vol/ $\mathrm{vol} / \mathrm{vol}$ ) as described previously (34). These extracts were used for analyzing total phospholipid content and individual phospholipids 
(PC, phosphatidylglycerol, and DSPC). Total phospholipid content was analyzed by measuring phosphorus (35). Part of the remaining extracts was subjected to thin-layer chromatography using silica $G$ plates (Adsorbosil Plus-1; Alltech, Deerfield, IL) and a system consisting of chloroform/ethanol/triethylamine/water (30:34:30:8) as the mobile phase to analyze PC and phosphatidylglycerol, whereas the other part was oxidized with osmium tetroxide $\left(\mathrm{OsO}_{4}\right)$ before subjection to thin-layer chromatography to analyze DSPC, the major surfactant component, as described previously (36). The chromatography plates were sprayed with $0.1 \%$ diphenylhexatriene in petroleum ether, and phospholipid standards were used to identify the corresponding bands, which were scraped off the plates and extracted into methanol, dried under a vacuum, and assayed for phosphorus content as described previously (35).

\section{Biochemical Analyses}

Activities of SOD, GSH-Px, and MPO, as well as MDA content of lung tissues, were measured using cell-free supernatants of right-lung homogenates by spectrophotometric (UV-1700; Shimadzu, Kyoto, Japan) analyses.

Tissue SOD activity was determined by a method involving spectrophotometric detection of formazan production at $550 \mathrm{~nm}$ by inhibition of nitroblue tetrazolium reduction with xanthine-xanthine oxidase used as a superoxide generator as described previously (37). Tissue GSH-Px activity was determined by the dithio-dinitrobenzoic acid method at the absorbance of $412 \mathrm{~nm}$ as described previously (38). Tissue MDA content was determined by a method that is based on the reaction of MDA with thiobarbituric acid at $95^{\circ} \mathrm{C}$ as described previously (39). Tissue MPO activity was determined by a method based on the reduction of 5-thio-2-nitrobenzoic acid to 5-5'-dithiobis (2-nitrobenzoic acid) by following the decrease of absorbance at $412 \mathrm{~nm}$ as described previously (40).

\section{Statistical Analyses}

Statistical analyses were performed using SPSS 16.0 (SPSS, Chicago, IL). A level of $P<0.05$ was chosen to represent statistical significance. Experimental group means and SEs were determined using descriptive statistics, and data were expressed as mean \pm SEM. Normal distributions of data were graphically examined with the ShapiroWilk test. Categorical variables were compared with the $\chi^{2}$ test. Immunohistochemical scores were assessed by the Kruskal-Wallis test. Treatment groups were compared using one-way ANOVA, and significance was determined using a post hoc Holm-Sidak test or Bonferroni's correction for multiple comparisons where applicable.

\section{STATEMENT OF FINANCIAL SUPPORT}

No financial assistance was received to support this work.

Disclosure: The authors declare no conflict of interest.

\section{REFERENCES}

1. Thomas W, Speer CP. Prevention and treatment of bronchopulmonary dysplasia: current status and future prospects. J Perinatol 2007;27: S26-32.

2. Gien J, Kinsella JP. Pathogenesis and treatment of bronchopulmonary dysplasia. Curr Opin Pediatr 2011;23:305-13.

3. Laughon MM, Smith PB, Bose C. Prevention of bronchopulmonary dysplasia. Semin Fetal Neonatal Med 2009;14:374-82.

4. Adibhatla RM, Hatcher JF. Cytidine 5'-diphosphocholine (CDPcholine) in stroke and other CNS disorders. Neurochem Res 2005;30: 15-23.

5. Zimmermann LJ, Janssen DJ, Tibboel D, Hamvas A, Carnielli VP. Surfactant metabolism in the neonate. Biol Neonate 2005;87:296-307.

6. Merrill JD, Ballard RA, Cnaan A, et al. Dysfunction of pulmonary surfactant in chronically ventilated premature infants. Pediatr Res 2004;56:91826.

7. Laughon M, Bose C, Moya F, et al.; Surfaxin Study Group. A pilot randomized, controlled trial of later treatment with a peptide-containing, synthetic surfactant for the prevention of bronchopulmonary dysplasia. Pediatrics 2009;123:89-96.
8. Colombo ML, Dogliani P, Raggi M. [Relation between citicoline and pulmonary surfactant. Recent acquisitions in the treatment of respiratory syndromes of the newborn]. Minerva Pediatr 1976;28: 2303-10.

9. Valls i Soler A, Sanjurjo P, Vazquez Cordero C. [Controlled study of the administration of CDP-choline to preterm newborn infants with respiratory distress syndrome]. An Esp Pediatr 1988;28:493-6.

10. Jobe AH, Ikegami M. Mechanisms initiating lung injury in the preterm. Early Hum Dev 1998;53:81-94.

11. Warner BB, Stuart LA, Papes RA, Wispé JR. Functional and pathological effects of prolonged hyperoxia in neonatal mice. Am J Physiol 1998;275(1 Pt 1):L110-7.

12. Mantell LL, Horowitz S, Davis JM, Kazzaz JA. Hyperoxia-induced cell death in the lung - the correlation of apoptosis, necrosis, and inflammation. Ann NY Acad Sci 1999;887:171-80.

13. McGrath-Morrow SA, Stahl J. Apoptosis in neonatal murine lung exposed to hyperoxia. Am J Respir Cell Mol Biol 2001;25:150-5.

14. Viscardi RM, Muhumuza CK, Rodriguez A, et al. Inflammatory markers in intrauterine and fetal blood and cerebrospinal fluid compartments are associated with adverse pulmonary and neurologic outcomes in preterm infants. Pediatr Res 2004;55:1009-17.

15. Paananen R, Husa AK, Vuolteenaho R, Herva R, Kaukola T, Hallman M. Blood cytokines during the perinatal period in very preterm infants: relationship of inflammatory response and bronchopulmonary dysplasia. J Pediatr 2009;154:39-43.e3.

16. Cansev M, Yilmaz MS, Ilcol YO, Hamurtekin E, Ulus IH. Cardiovascular effects of CDP-choline and its metabolites: involvement of peripheral autonomic nervous system. Eur J Pharmacol 2007;577:129-42.

17. Wurtman RJ, Cansev M, Ulus IH. Choline and its products acetylcholine and phosphatidylcholine. In: Lajtha A, ed. Neural Lipids, Handbook of Neurochemistry and Molecular Neurobiology. Berlin, Heidelberg: Springer-Verlag, 2009:443-501.

18. Parrish WR, Rosas-Ballina M, Gallowitsch-Puerta M, et al. Modulation of TNF release by choline requires alpha7 subunit nicotinic acetylcholine receptor-mediated signaling. Mol Med 2008;14:567-74.

19. Ilcol YO, Yilmaz Z, Ulus IH. Endotoxin alters serum-free choline and phospholipid-bound choline concentrations, and choline administration attenuates endotoxin-induced organ injury in dogs. Shock 2005;24:28893.

20. Holmes-McNary MQ, Baldwin AS Jr, Zeisel SH. Opposing regulation of choline deficiency-induced apoptosis by $\mathrm{p} 53$ and nuclear factor kappaB. J Biol Chem 2001;276:41197-204.

21. Kolahian S, Gosens R. Cholinergic regulation of airway inflammation and remodelling. J Allergy (Cairo) 2012;2012:681258.

22. Tracey KJ. Physiology and immunology of the cholinergic antiinflammatory pathway. J Clin Invest 2007;117:289-96.

23. de Jonge WJ, Ulloa L. The alpha7 nicotinic acetylcholine receptor as a pharmacological target for inflammation. Br J Pharmacol 2007;151: 915-29.

24. Blanchet MR, Israël-Assayag E, Cormier Y. Modulation of airway inflammation and resistance in mice by a nicotinic receptor agonist. Eur Respir J 2005;26:21-7.

25. Cansev M, Ilcol YO, Yilmaz MS, Hamurtekin E, Ulus IH. Choline, CDPcholine or phosphocholine increases plasma glucagon in rats: involvement of the peripheral autonomic nervous system. Eur J Pharmacol 2008;589:315-22.

26. Savani RC, Godinez RI, Godinez MH, et al. Respiratory distress after intratracheal bleomycin: selective deficiency of surfactant proteins B and C. Am J Physiol Lung Cell Mol Physiol 2001;281:L685-96.

27. Ballard PL, Gonzales LW, Godinez RI, et al. Surfactant composition and function in a primate model of infant chronic lung disease: effects of inhaled nitric oxide. Pediatr Res 2006;59:157-62.

28. Katz LA, Klein JM. Repeat surfactant therapy for postsurfactant slump. J Perinatol 2006;26:414-22.

29. Cogo PE, Zimmermann LJ, Pesavento R, et al. Surfactant kinetics in preterm infants on mechanical ventilation who did and did not develop bronchopulmonary dysplasia. Crit Care Med 2003;31:1532-8. 
30. Cogo PE, Toffolo GM, Gucciardi A, Benetazzo A, Cobelli C, Carnielli VP. Surfactant disaturated phosphatidylcholine kinetics in infants with bronchopulmonary dysplasia measured with stable isotopes and a twocompartment model. J Appl Physiol 2005;99:323-9.

31. Ozdulger A, Cinel I, Koksel O, et al. The protective effect of N-acetylcysteine on apoptotic lung injury in cecal ligation and puncture-induced sepsis model. Shock 2003;19:366-72.

32. Askenazi SS, Perlman M. Pulmonary hypoplasia: lung weight and radial alveolar count as criteria of diagnosis. Arch Dis Child 1979;54:614-8.

33. Bouadma L, Schortgen F, Ricard JD, Martet G, Dreyfuss D, Saumon G. Ventilation strategy affects cytokine release after mesenteric ischemiareperfusion in rats. Crit Care Med 2004;32:1563-9.

34. Folch J, Lees M, Sloane Stanley GH. A simple method for the isolation and purification of total lipides from animal tissues. J Biol Chem 1957;226:497509.
35. Svanborg A, Svennerholm L. Plasma total lipids, cholesterol, triglycerides, phospholipids and free fatty acids in a healthy Scandinavian population. Acta Med Scand 1961;169: 43-9.

36. Mason RJ, Nellenbogen J, Clements JA. Isolation of disaturated phosphatidylcholine with osmium tetroxide. J Lipid Res 1976;17:281-4.

37. Sun Y, Oberley LW, Li Y. A simple method for clinical assay of superoxide dismutase. Clin Chem 1988;34:497-500.

38. Paglia DE, Valentine WN. Studies on the quantitative and qualitative characterization of erythrocyte glutathione peroxidase. J Lab Clin Med 1967;70:158-69.

39. Draper HH, Hadley M. Malondialdehyde determination as index of lipid peroxidation. Meth Enzymol 1990;186:421-31.

40. Weiss SJ, Klein R, Slivka A, Wei M. Chlorination of taurine by human neutrophils. Evidence for hypochlorous acid generation. J Clin Invest 1982;70:598-607. 